\title{
Cinnamic-Derived Acids Significantly Affect Fusarium graminearum Growth and In Vitro Synthesis of Type B Trichothecenes
}

\author{
Nadia Ponts, Laetitia Pinson-Gadais, Anne-Laure Boutigny, Christian Barreau, and Florence Richard-Forget
}

First author: Department of Cell Biology and Neuroscience, 900 University Avenue, University of California-Riverside 92521; second, third, and fifth authors: INRA, Centre de Bordeaux, UR1264 MycSA, 71 Avenue Edouard Bourleaux, BP81, 33883 Villenave d'Ornon Cedex, France; and fourth author: CNRS, INRA, Centre de Bordeaux, UR1264 MycSA, 71 Avenue Edouard Bourleaux, BP81, 33883 Villenave d'Ornon Cedex, France.

Accepted for publication 5 March 2011.

\begin{abstract}
Ponts, N., Pinson-Gadais, L., Boutigny, A. L., Barreau, C., and RichardForget, F. 2011. Cinnamic acids significantly affect Fusarium graminearum growth and in vitro production of type $\mathrm{B}$ trichothecenes. Phytopathology 101:929-934.

The impact of five phenolic acids (ferulic, coumaric, caffeic, syringic, and $p$-hydroxybenzoic acids) on fungal growth and type B trichothecene production by four strains of Fusarium graminearum was investigated. All five phenolic acids inhibited growth but the degree of inhibition

and peptone cultures was enhanced in the presence of ferulic and coumaric acids but was reduced in the presence of $p$-hydroxybenzoic acid. This modulation was shown to correlate with a regulation of TRI5 transcription. In this study, addition of phenolic acids with greater antioxidant properties resulted in a higher toxin accumulation, indicating that the modulation of toxin accumulation may be linked to the antioxidant properties of the phenolic acids. These data suggest that, in planta, different compositions in phenolic acids of kernels from various cultivars may reflect different degrees of sensitivity to "mycotoxinogenesis."
\end{abstract} varied between strains. Our results suggested that the more lipophilic phenolic acids are, the higher is the effect they have on growth. Toxin accumulation in phenolic acid-supplemented liquid glucose, yeast extract,
Additional keywords: ferulic acid.
Fusarium graminearum is a prevalent phytopathogen on cereals. In planta, it induces Fusarium head blight, also known as Fusarium scab, of small grain cereals and maize ear rot, two diseases with a huge economic impact in many cereal-growing areas around the world. Furthermore, some $F$. graminearum strains can produce deoxynivalenol (DON) and its 3- and 15acetylated forms (3ADON and $15 \mathrm{ADON}$, respectively) that belong to the type B trichothecenes (TCTB) family of mycotoxins. DON toxicity has been well documented (12) and represents a real public health problem.

TCTB are highly stable. Because these toxins are resistant to biodegradation and food processing and there is no suitable detoxification procedure available at present, the occurrence of TCTB in kernels cannot be reduced by post-harvest treatments. In June 2005, maximum DON contamination levels in cereals and corn food and feed were set in Europe (EC No856/2005). These levels were revised in July 2007 (EC No1126/2007).

Implementing strategies to provide an efficient management of the mycotoxic risk requires a better understanding of events leading to TCTB accumulation in kernels. A number of strategies for reducing TCTB concentrations in wheat and maize ears are currently under investigation (1) and among the most promising is the identification of naturally occurring plant compounds able to inhibit TCTB biosynthesis.

The TCTB biosynthesis pathway is fairly well understood (24) as a result of the enhanced effort at identifying biosynthetic steps and genes involved in mycotoxin production developed during the last 10 years. Nearly all the genes implicated in the TCTB biosynthesis pathway, commonly named TRI genes, have been

Corresponding author: F. Richard-Forget; E-mail address: fforget@ bordeaux.inra.fr

doi:10.1094/PHYTO-09-10-0230

(C) 2011 The American Phytopathological Society identified. Most of them are localized in one genomic locus which is referred as the "FgTRI5 cluster". Among the clustered genes, TRI5 plays a crucial role in TCTB production because it encodes the trichodiene synthase that catalyzes the first step of the TCTB biosynthesis pathway (24). One of the key challenges that research on $F$. graminearum now has to answer is the elucidation of the effects of environmental factors on initiation or repression of toxin biosynthesis. Knowledge concerning the factors that could control TCTB production has been recently updated (10). The main consistent data published recently concern the effects of carbon and nitrogen sources (20) and the identification of specific amines such as agmatin and putrescine as potent inducers of trichothecenes B biosynthesis (13). A previous work has also demonstrated that pro-oxidant compounds significantly modulated $\mathrm{DON}$ and $15 \mathrm{ADON}$ biosynthesis by $F$. graminearum. In particular, $\mathrm{H}_{2} \mathrm{O}_{2}$ was shown to have a long-term activating effect on TCTB accumulation while the kinetics of fungal biomass accumulation was not affected (32). This event was indicated to be linked to a general upregulation of some TRI genes involved in the DON and 15ADON biosynthesis pathway $(27,31)$. Because plant metabolites with pro-oxidant properties have a strong effect on mycotoxin biosynthesis, we could reasonably suppose that plant secondary metabolites with antioxidant properties may also interfere with mycotoxin biosynthesis. Phenolic acids are typical cereal and corn compounds described as potent antioxidants $(7,21,36)$. In corn kernels, monomers and dimers of ferulic acid are largely predominant (5). Phenolic compounds have been extensively studied with regard to Fusarium head blight and have been shown to be involved in the disease resistance $(7,17)$. Moreover, the content in phenolic compounds was shown to be higher in resistant maize silks infected with $F$. graminearum than in sensitive Fusarium spp.-infected maize silks (33). Furthermore, some phenolic compounds have been reported as fungistatic and inhibitors of toxin production. For example, caffeic acid and 
ferulic acid can reduce fumonisin $\mathrm{B}_{1}$ production by $F$. verticillioides (6). Aflatoxin production by Aspergillus spp. is reduced by ferulic acid, syringaldehyde and sinapinic acid (18), and caffeic acid (22). Finally, previous studies have also suggested that caffeic, ferulic, p-coumaric, and 3-hydroxybenzoic acids were able to act on $15 \mathrm{ADON}$ production by $F$. graminearum $(5,9)$. However, these reports required to be clarified, and the mechanisms by which phenolic acids could affect trichothecenes production remained to be elucidated.

Thus, the main objective of this investigation was to clarify the effect of phenolic acids on TCTB accumulation. Five phenolic acids (ferulic, $p$-coumaric, caffeic, syringic, and $p$-hydroxybenzoic acids) were considered. These phenolic acids were selected because of their relative abundance in cereals and their well-known antioxidant properties. Their effects on F. graminearum growth and on DON and 15ADON accumulation were investigated and attempts to establish structure-modulating efficiency relationships were achieved.

\section{MATERIALS AND METHODS}

F. graminearum strains. Four strains of $F$. graminearum were used: F. graminearum strain CBS185.32 (Centraal Bureau voor Shimmelkulturen, The Netherlands), strain 245AP4 (HortResearch and AgResearch, New Zealand), and strains INRA 159 and INRA 202 (collection of INRA MycSA, France). They all produce predominantly $15 \mathrm{ADON}$ and a lower amount of DON. Usually, INRA 159 produces very low amounts of toxins $(<1 \mu \mathrm{g}$ of toxin per gram of dry fungal biomass after 15 days of incubation in glucose, yeast extract, and peptone (GYEP) medium at $\left.25^{\circ} \mathrm{C}\right)(26)$ while CBS185.32 and INRA 202 are strong toxinproducing strains $(>30 \mu \mathrm{g}$ of toxin per gram of dry fungal biomass after 15 days of incubation in GYEP medium at $25^{\circ} \mathrm{C}$ ); the strain $245 \mathrm{AP} 4$ produces average amounts of toxins (close to $10 \mu \mathrm{g}$ of toxin per gram of dry fungal biomass after 15 days of incubation in GYEP medium at $25^{\circ} \mathrm{C}$ ). Strains were maintained on potato dextrose agar (PDA) (Sigma-Aldrich, St. Louis) slants at $4^{\circ} \mathrm{C}$. When inocula were required, spore suspensions were prepared by adding sterile distilled water to the slants followed by gentle shaking. Spores concentrations were evaluated using a Thoma cell.

Effect of phenolic acids on the growth of $F$. graminearum CBS185.32, INRA 159, INRA 202, and 245AP4. Growth inhibition tests were performed on PDA medium in petri dishes (diameter $=9 \mathrm{~cm}$ ) inoculated at the centre with $5 \mu$ l of spore suspension at $10^{3}$ spores $\mathrm{ml}^{-1}$. PDA media were supplemented with ferulic, $p$-coumaric, caffeic, syringic, or $p$-hydroxybenzoic acid (Sigma-Aldrich) at 2, 3, 4, 5, or $6 \mathrm{mmol} \mathrm{liter}^{-1}$. For each condition, plates (in triplicate) were incubated at $25^{\circ} \mathrm{C}$ for 5 days; that is to say, until $80 \%$ of the petri dish area was colonized in the control dishes. In each plate, the colony diameter value was given as the average of two perpendicular diameter length measurements (in centimeters). Growth inhibition values (GIVs) were expressed as the average percentage of the petri dish covered by mycelium in the control dishes minus the percentage overgrown by the fungus in the treated dishes. For each phenolic acid, the relation GIV $=f([$ phenolic acid $])$ was plotted and concentration that inhibits $50 \%$ of growth $\left(\mathrm{IC}_{50}\right)$ was determined by linear regression for GIV $=50 \%$.

Estimation of the relative lipophilicities of the tested phenolic acids. Samples were prepared and analyzed by reverse-phase highperformance liquid chromatography (HPLC) using the procedure of Ponts et al. (32). HPLC analyses were monitored at $280 \mathrm{~nm}$. The retention times of the phenolic acids were compared and classified in order to evaluate their relative degrees of lipophilicity.

Effect of phenolic acids on DON and 15ADON accumulation in liquid cultures of $F$. graminearum CBS185.32, INRA 159, INRA 202, and 245AP4. The liquid medium GYEP (26) was used throughout the study. The medium was supplemented before inoculation with ferulic, $p$-coumaric, caffeic, syringic, or $p$-hydroxybenzoic acid. The final concentration of each phenolic acid was 0.5 mmol liter ${ }^{-1}$. Then, $100 \mathrm{ml}$ of GYEP in 500-ml Erlenmeyer flasks was inoculated with $10^{6}$ spores and incubated at $25^{\circ} \mathrm{C}$ and $150 \mathrm{rpm}$ into darkness in a Multitron incubator shaker (INFORS AG, Switzerland). Cultures were stopped by filtering under vacuum through Whatman paper number 4 . The filtrates were stored at $-20^{\circ} \mathrm{C}$. TCTB levels were determined in culture filtrates, and fungal biomass production was determined by weighting the mycelia after $48 \mathrm{~h}$ of freeze drying. Each culture condition was repeated in triplicate.

TCTB were analyzed according to the protocol described by Ponts et al. (32). Briefly, $15 \mathrm{ml}$ of filtrates was extracted with $30 \mathrm{ml}$ of ethyl acetate; $20 \mathrm{ml}$ of the organic phase was evaporated to dryness at $70^{\circ} \mathrm{C}$ under a nitrogen stream. Dried samples were resuspended in $200 \mu \mathrm{l}$ of methanol/water $(50 \%$, vol/vol) before analysis by HPLC-DAD. Quantification was performed by using external calibration with DON and 15ADON standard solutions prepared from commercial pure powders (Sigma-Aldrich). Toxin levels were expressed in micrograms of toxin per gram of dry fungal biomass divided by the toxin yield measurement after 15 days of control culture. Because effects of phenolic acids on DON and 15ADON accumulation were consistent for all tested strains, DON and 15ADON yields were summed without distinguishing both chemical species (noted as DON+15ADON). Data are expressed as arithmetic mean values \pm standard deviation. Values were compared by computing standard Student's $t$ test. The $P$ value 0.05 was chosen as the point of statistical significance.

Extraction of total RNA and preparation of cDNA. Fresh mycelium (200 mg) of 4-day-old cultures was grounded in $1 \mathrm{ml}$ of TRIzol reagent (Invitrogen Life Technologies) using the TissueLyser System (Qiagen). Total RNA was extracted using the TRIzol reagent manufacturer's instructions. The quality of the RNA was verified by agarose gel electrophoresis. Total RNA $(3 \mu \mathrm{g})$ was reverse transcribed using the Superscript II FirstStrand Synthesis System (Invitrogen Life Technologies).

Real-time polymerase chain reaction analysis. Abundance of the transcripts of the gene TRI5 was evaluated using $3 \mu \mathrm{l}$ of each cDNA solution diluted 10 times by real-time polymerase chain reaction (PCR) using LightCycler FastStart DNA Master SYBR Green I and a LightCycler real-time detector (Roche Applied Science). Expression of $\beta$-tubulin was used as an endogenous reference. Analyses were performed in triplicate. Primers used to amplify these genes were Tri5-F GACCCTAAGCGACTACAG and Tri5-R GTGCTACGGATAAGGTTC (35) for TRI5 amplification, and $\beta t 2 a$ GGTAACCAAATCGGTGCTGCTTTC (15) and $\beta t u b$-R536 ( $\beta t$-2) GATTGACCGAAAACGAAGTTG (30) for $\beta$-tubulin amplification. They were used at a final concentration of $500 \mathrm{nM} . \mathrm{MgCl}_{2}$ was added to the PCR mix to achieve a final concentration of $4 \mathrm{mM}$. Amplification was performed in triplicate for each of the three biological replications.

cDNA sample solutions (10 $\mu \mathrm{l}$ each) were mixed together to prepare a standard mixture to be used to determine PCR efficiencies $(E)$ by external calibration, according to a previously described protocol (31). E was calculated using the LightCycler Software (version 4.05; Roche Applied Science). PCR efficiencies and standard errors obtained for reference and TRI5 genes were $1.93 \pm 0.03$ and $1.97 \pm 0.02$, respectively. All cDNA samples were tested for residual genomic DNA (31). The absence of genomic DNA was verified by running both melting curves and agarose gels analyses on the final PCR products. The same procedure was followed to check the absence of nonspecific PCR amplification or primer dimers formation.

Real-time PCR data analysis. Data were analyzed according to the procedure of Ponts et al. (31). The crossing point (Cp) values obtained experimentally (mean value of three replicates) for $\beta$ - 
tubulin were compared in order to verify the stability of $\beta$-tubulin expression in the presence of phenolic acids. When the cultures were treated with either ferulic or $p$-hydroxybenzoic acid, data were analyzed with the REST software. The expression levels of TRI5, normalized to reference gene expression, are expressed as a regulation factor in the treated condition relative to the control,

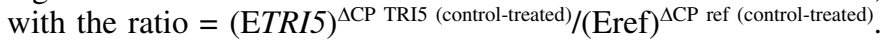
$P=0.001$ was chosen as the statistical point throughout.

\section{RESULTS}

Effect of phenolic acids on the growth of $F$. graminearum CBS185.32, INRA 159, INRA 202, and 245AP4. When considering the CBS185.32 strain, phenolic acid $\mathrm{IC}_{50}$ values ranged from $1.7 \mathrm{mmol} \mathrm{liter}^{-1}$ to $>15 \mathrm{mmol} \mathrm{liter}^{-1}$ (Table 1 ). The lower the $\mathrm{IC}_{50}$ value, the higher the fungicidal properties of the phenolic acid under consideration. Thus, ferulic acid can be considered to be the most toxic compound and $p$-hydroxybenzoic acid the least toxic one (little growth inhibition). Similar experiments were performed on $F$. graminearum INRA $159, F$. graminearum INRA 202, and F. graminearum 245AP4; results are reported in Table 1. It clearly appears that ranking of the fungicidal activity of the phenolic acids is independent of the Fusarium strain tested. Ferulic acid is the most toxic compound, followed by $p$-coumaric acid, syringic acid, caffeic acid, and $p$-hydroxybenzoic acid.

Lipophilic characteristics of phenolic acids and their effects on growth of $\boldsymbol{F}$. graminearum. Because previous studies indicated that fungitoxic properties of phenolic compounds may primary depend on their lipophilic properties (16), it was investigated whether our experimental $\mathrm{IC}_{50}$ values of phenolic acids

TABLE 1. Experimental concentration that inhibits $50 \%$ of growth $\left(\mathrm{IC}_{50}\right)$ values of five phenolic acids measured on the growth of Fusarium graminearum CBS185.32, INRA 202, 245AP4, and INRA 159

\begin{tabular}{lcccc}
\hline & \multicolumn{4}{c}{ Experimental $\mathrm{IC}_{50}\left(\mathrm{mmol} \mathrm{liter}^{-1}\right)$} \\
\cline { 2 - 5 } Phenolic acid & CBS 185.32 & INRA 202 & 245AP4 & INRA159 \\
\hline Ferulic acid & 1.7 & 1.8 & 2.2 & 0.7 \\
$p$-Coumaric acid & 4.1 & 4.2 & 4.1 & 1.0 \\
Syringic acid & 5.2 & 5.7 & 6.2 & 3.5 \\
Caffeic acid & 6.6 & 6.4 & 7.1 & 4.0 \\
$p$-Hydroxybenzoic acid & $>15.0^{\mathrm{a}}$ & 10.3 & 8.4 & 6.6 \\
\hline
\end{tabular}

${ }^{a}$ Little growth inhibition.

TABLE 2. Retention times (in minutes) measured when separation of phenolic acids is performed by reverse-phase high-performance liquid chromatography following four different methods ${ }^{\mathrm{a}}$

\begin{tabular}{lcccc}
\hline Phenolic acid & $\begin{array}{c}\text { Bolarinwa and } \\
\text { Linseisen (8) }\end{array}$ & $\begin{array}{c}\text { Jandera } \\
\text { et al. } \\
(19)\end{array}$ & $\begin{array}{c}\text { Antolovich } \\
\text { et al. (3) }\end{array}$ & $\begin{array}{c}\text { TCTB } \\
\text { analysis } \\
\text { method }^{\text {b }}\end{array}$ \\
\hline Ferulic acid & 51.0 & 53.3 & 14.0 & 11.0 \\
$p$-Coumaric acid & 42.3 & 37.2 & 13.8 & 10.0 \\
Syringic acid & 36.5 & 25.0 & 12.3 & 8.1 \\
Caffeic acid & 30.9 & 21.3 & 12.2 & 7.6 \\
$p$-Hydroxybenzoic acid & ND & ND & ND & 6.7
\end{tabular}

${ }^{\mathrm{a}} \mathrm{ND}=$ not determined.

${ }^{b}$ TCTB $=$ type B trichothecenes; described in Materials and Methods. could be linked to their degrees of lipophilicity. Lipophilicities of phenolic acids were scored using their retention times when separated by the reverse-phase HPLC method (see Materials and Methods). Our results were compared with retention times obtained with other reverse-phase HPLC methods $(3,8,19)$. All data are consistent (Table 2), and a classification of phenolic acids according to their decreasing degrees of lipophilicity can be established: phenolic acids with higher retention times are more lipophilic. Ferulic acid is the most lipophilic compound, followed by $p$-coumaric acid, syringic acid, caffeic acid, and $p$-hydroxybenzoic acid. For each $F$. graminearum strain and each reversephase HPLC method, Pearson's correlation coefficients were calculated between $\mathrm{IC}_{50}$ values of phenolic acids and their retention times (Table 3). Depending on the HPLC method that was used and the $F$. graminearum strain considered, correlation coefficients ranged from -0.8849 , when retention times were determined with the method from Antolovich et al. (3) and for strain CBS185.32, up to -0.9988 , when retention times were determined with the method from Jandera et al. (19) for isolate INRA 202. These high values support the initial hypothesis that more lipophilic phenolic acids have greater fungistatic efficiency.

Effect of phenolic acids on DON and 15ADON accumulation in liquid cultures of $F$. graminearum CBS185.32, INRA 159, INRA 202, and 245AP4. Effects of ferulic, $p$-coumaric, syringic, caffeic, and $p$-hydroxybenzoic acids on DON and 15ADON accumulation were first studied at $0.5 \mathrm{mmol} \mathrm{liter}^{-1}$ in liquid cultures of $F$. graminearum CBS185.32. Cultures were grown for 4,11 , and 15 days. Whatever the considered phenolic acid, a 0.5 mmol liter ${ }^{-1}$ concentration is much lower than the $\mathrm{IC}_{50}$ of the compounds, and fungal growth after 11 and 15 days in GYEP culture was not affected (data not shown). As a reference, the effect of buthylhydroxytoluene (BHT) at $0.5 \mathrm{mmol}^{\text {liter }}{ }^{-1}$ after 11 days of culture was also determined because of its efficient antioxidant properties. BHT at $0.5 \mathrm{mmol} \mathrm{liter}^{-1}$ did not affect biomass accumulation in GYEP cultures of $F$. graminearum CBS 185.32 (data not shown). After 11 days of culture, DON+15ADON accumulation was 24 times higher in the presence of BHT (Fig. 1). Ferulic acid and $p$-coumaric acid both activated DON+15ADON accumulation (11 and 20 times, respectively). Caffeic acid seemed also to enhance toxin accumulation but high variance of toxin yields in caffeic acid-supplemented cultures strongly lowers the significance of this observation (biological variation). Syringic acid supplementation had no effect on DON+15ADON accumulation. Only $p$-hydroxybenzoic acid had a significant inhibitory effect, with three times less toxin accumulating in supplemented cultures than in control cultures $(P=0.02)$. These trends remain observable after 15 days of culture but with weaker intensities (Fig. 1). The impact of

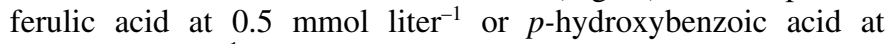
$0.5 \mathrm{mmol}$ liter $^{-1}$ on the levels of TRI5 gene expression was evaluated in 4-day-old cultures of $F$. graminearum (CBS 185.32). Four-day-old cultures were chosen for investigation because it has been previously demonstrated that transcripts of the TRI5 gene reach their highest level between 3 and 4 days of culture in GYEP media to then attain a low residual level $(13,31)$. Analysis of the $\beta$-tubulin $\mathrm{Cp}$ values measured in control and phenolic-acidsupplemented cultures showed that levels of $\beta$-tubulin mRNA in

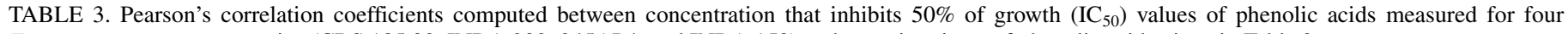
Fusarium graminearum strains (CBS 185.32, INRA 202, 245AP4, and INRA 159) and retention times of phenolic acids given in Table 2

\begin{tabular}{|c|c|c|c|c|}
\hline HPLC method ${ }^{\mathrm{a}}$ & CBS185.32 & INRA202 & 245AP4 & INRA159 \\
\hline Bolarinwa and Linseisen (8) & -0.9976 & -0.9898 & -0.9920 & -0.9241 \\
\hline Jandera et al. (19) & -0.9840 & -0.9988 & -0.9921 & -0.9209 \\
\hline Antolovich et al. (3) & -0.8849 & -0.9083 & -0.9535 & -0.9968 \\
\hline TCTB analysis method & -0.9580 & -0.9431 & -0.9961 & -0.9620 \\
\hline
\end{tabular}

a HPLC = high-performance liquid chromatography; TCTB = type B trichothecenes; described in Materials and Methods. 
total RNA were not significantly affected by the treatment, supporting the use of $\beta$-tubulin as a reference gene. The expression values of TRI5 relative to the reference gene were expressed as a up- or downregulation factor in the treated conditions relative to the control. The ferulic acid treatment was shown to induce a significant upregulation of TRI5 with a regulation factor of $4.3 \pm$ $1.3\left(\mathrm{Cp}_{\text {TRIS }}=26.9 \pm 0.25\right.$ in control cultures and $25.2 \pm 0.18$ in ferulic-acid-treated cultures) in accordance with the enhancement of the DON+15ADON accumulation that was reported above. A weak downregulation of TRI5 (regulation factor of $1.82 \pm 0.25$ ) that was, however, not significant was observed in $p$-hydroxybenzoic-acid-supplemented cultures. These data indicated that the effect of phenolic acids on TCTB accumulation could be linked to a regulation of the expression of the TRI5 gene involved in the trichothecenes biosynthetic pathway.

Impact of phenolic acids on TCTB production was investigated in 15-day-old cultures of $F$. graminearum INRA 159, INRA 202, and 245AP4. Whatever the considered strain and phenolic acid,

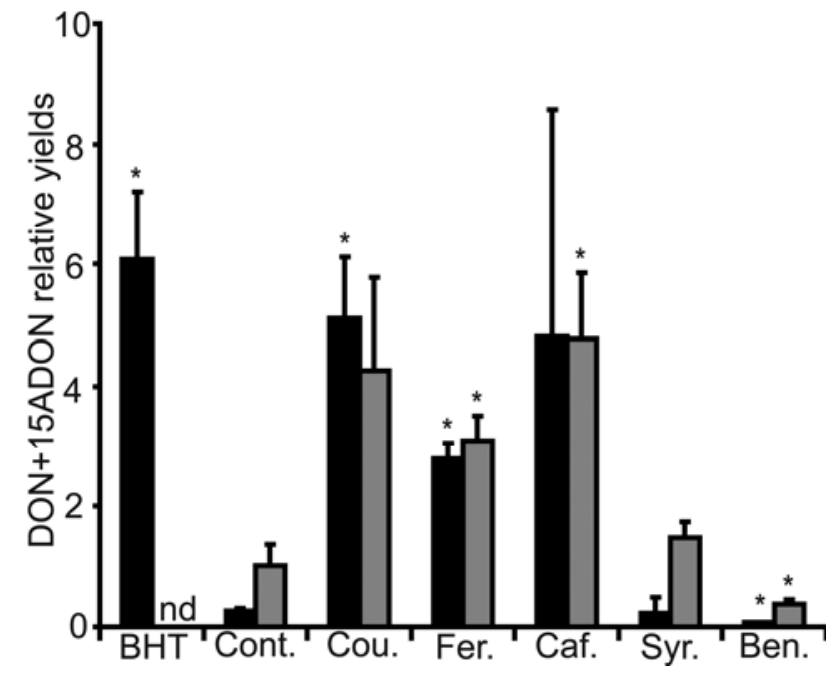

Fig. 1. Effects of five phenolic acids or buthylhydroxytoluene (BHT) $(0.5 \mathrm{mmol}$ liter $^{-1}$ ) on deoxynivalenol (DON) plus15-acetylated DON (DON+15ADON) accumulation in 11-day-old (black bars) and 15-day-old (gray bars) glucose, yeast extract, and peptone cultures of Fusarium graminearum CBS185.32. Cont. $=$ control, Cou. $=p$-coumaric acid, Fer. $=$ ferulic acid, Caf. $=$ caffeic acid, Syr. $=$ syringic acid, Ben.$=p$-hydroxybenzoic acid, nd $=$ not determined. Data obtained from three biological replications were normalized relative to the average DON+15ADON yield in 15-day-old control cultures $(=50 \mu \mathrm{g}$ of DON and 15ADON per gram of dry biomass). Asterisk (*) indicates a significant difference when compared with the corresponding control $(P \leq 0.05)$. fungal biomass production was not affected by the $0.5 \mathrm{mmol}$ liter $^{-1}$ concentration used (data not shown). One of the tested strains, INRA 159 , usually produces very low amounts of toxins $(<1 \mu \mathrm{g}$ of toxin per gram of dry fungal biomass). In the presence of ferulic acid, toxin production by this $F$. graminearum strain seems to be slightly enhanced whereas, in the presence of $p$-hydroxybenzoic acid, it seems to be slightly reduced. However, none of the tested phenolic acid had a quantifiable effect (data not shown). With regard to $F$. graminearum strains 245AP4 (Fig. 2A) and INRA 202 (Fig. 2B), DON+15ADON accumulation was significantly enhanced in the presence of $p$-coumaric acid ( 2 times in the case of INRA 202 and 44 times in the case of 245AP4) and ferulic acid ( 2 times in the case of INRA 202 and 100 times in the case of 245AP4). p-Hydroxybenzoic acid significantly inhibited DON+15ADON accumulation for both strains. Finally, opposite effects of caffeic acid and syringic acid were observed as far as these two $F$. graminearum strains are concerned: caffeic acid and syringic acid enhanced toxin production by $F$. graminearum strain 245AP4 but inhibited it with regard to strain 202.

Antioxidant characteristics of phenolic acids and their effect on DON and 15ADON accumulation in liquid cultures of $\boldsymbol{F}$. graminearum. Because previous studies have suggested that DON and 15ADON accumulation could be significantly affected by the oxidative status of the medium (32), it was investigated whether DON+15ADON yields in phenolic-acid-supplemented cultures could be linked to the antioxidant properties of the compounds. To assess antioxidant capacities of phenolic compounds from literature data is difficult, because results largely depend on the experimental protocol that is used. Nevertheless, authors' classifications of phenolic acids according to their decreasing antioxidant capacities are consistent $(21,34)$. The highest antioxidant capacity is measured for $p$-coumaric acid, followed by ferulic acid, caffeic acid, syringic acid, and then $p$ hydroxybenzoic acid. The antioxidant capacities of these five phenolic acids, valued using the vitamin $\mathrm{C}$ equivalent antioxidant capacity (VCEAC) method (21), are presented in Table 4. Pearson's correlation coefficients were computed between the VCEAC values of phenolic acids and DON+15ADON yields in 11 - and 15-day-old cultures of $F$. graminearum CBS185.32. For the 11-day-old cultures, correlation coefficient was 0.74 . It reached 0.76 when the 15 -day-old cultures were considered. Correlation coefficients were close to 0.70 and 0.86 with regard to 15-day-old cultures of the strains INRA 202 and 245AP4, respectively. These correlation coefficient values support the hypothesis that effect of phenolic acids on DON and 15ADON accumulation may be tightly linked with their antioxidant capacities: the more antioxidant the supplemented phenolic acid is, the more DON and $15 \mathrm{ADON}$ accumulate in the culture.
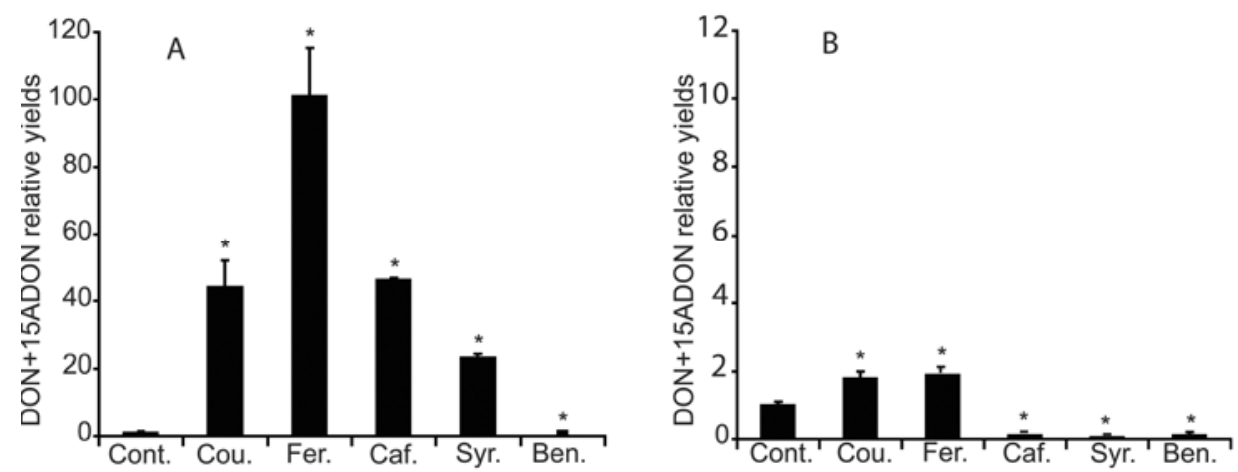

Fig. 2. Effects of five phenolic acids $\left(0.5 \mathrm{mmol}^{\text {liter }}{ }^{-1}\right)$ on deoxynivalenol (DON) plus15-acetylated DON (DON+15ADON) accumulation in 15-day-old glucose, yeast extract, and peptone cultures of Fusarium graminearum A, 245AP4 and B, INRA 202. Cont. = control, Cou. = $p$-coumaric acid, Fer. $=$ ferulic acid, Caf. $=$ caffeic acid, Syr. $=$ syringic acid, Ben. $=p$-hydroxybenzoic acid. For each strain, data obtained from three biological replications were normalized relative to the average DON+15ADON yield in corresponding control cultures (INRA 202: $70 \mu \mathrm{g}$ of DON+15ADON per gram of dry fungal biomass; 245AP4: $8 \mu \mathrm{g}$ of DON+15ADON per gram of dry fungal biomass). Asterisk $(*)$ indicates a significant difference when compared with the corresponding control $(P \leq 0.05)$. 


\section{DISCUSSION}

According to our results, all the tested phenolic acids inhibited $50 \%$ of $F$. graminearum radial growth at concentrations ranging from $0.7 \mathrm{mmol} \mathrm{liter}^{-1}$ (ferulic acid) to $>15 \mathrm{mmol} \mathrm{liter}^{-1}$ ( $p$-hydroxybenzoic acid). Although these concentrations were dependent on the F. graminearum strain that was used, the classification of phenolic acids according to their increasing $\mathrm{IC}_{50}$ values was consistent for all strains. The most toxic compound was ferulic acid, then $p$-coumaric acid, syringic acid, caffeic acid, and $p$ hydroxybenzoic acid. Ferulic and $p$-coumaric acid are both derived from cinnamic acid, while $p$-hydroxybenzoic and syringic acid are derived from benzoic acid. Therefore, our data suggest that cinnamic-derived phenolic acids are roughly more toxic than benzoic acid-derived ones. These data are consistent with those of previous tests on Aspergillus spp. growth (11) and on F. verticillioides and $F$. proliferatum growth (6). When $\mathrm{IC}_{50}$ values of phenolic acids were plotted against their lipophilicity scores, high correlation coefficients were obtained. This result suggests a strong link between toxicity of phenolic acids and lipophilic properties: the best fungistatic effect seems to be obtained for the compounds that are highly lipophilic. These data are in accordance with previous results $(23,28)$ that supported the hypothesis that lipophilic properties of phenol compounds are primary factors for their antimicrobial action.

As far as the effect of phenolic acids on TCTB accumulation is concerned, two previous reports indicated that ferulic acid supplementation led to a reduction of $15 \mathrm{ADON}$ production by $F$. graminearum $(7,9)$. This is apparently in contrast to our results that suggest that, in GYEP liquid cultures of $F$. graminearum, cinnamic-derived acids, including ferulic acid, are enhancers of DON+15ADON accumulation while benzoic-derived phenolic acids are more likely inhibitors. Two main hypotheses can be raised to explain these apparently contradictory results. The first one concerns the culture medium. In our experiments, contrary to the medium used by Bily (7) and Boutigny et al. (9), the GYEP medium was a very rich medium that also contains yeast extract, the organic compounds of which could interfere with the effect of phenolic acids. The high glucose concentration $\left(50 \mathrm{~g} \mathrm{liter}^{-1}\right)$ may also influence phenolic acids activity, as reported previously (4). The second hypothesis concerns the time of ferulic acid addition. In our experiments, ferulic acid was supplemented at the same time point as the seeding of the culture and was present during the germination stage; in Bily's experiments (7), ferulic acid was added during the stationary phase of fungal growth. Previous data have reported that the $F$. graminearum toxin-producing potential was closely linked to the biochemical environment surrounding its spores during germination (32).

Statistic analysis of our data has led to high and positive correlation coefficients between antioxidant capacities of the tested phenolic acids and their effect on DON and 15ADON accumulation ( 0.70 to 0.85 , depending on the $F$. graminearum strain under consideration). These observations suggest that the antioxidant capacities of the phenolic acids are tightly linked with their effect on DON+15ADON accumulation: the more antioxidant the supplemented phenolic acid is, the more DON and $15 \mathrm{ADON}$ accumulate in the culture. Because cinnamic acids were shown to inhibit the breakdown of hydrogen peroxide by fungi

TABLE 4. Vitamin C equivalent antioxidant capacity (VCEAC) value of five phenolic acids

\begin{tabular}{lc}
\hline Phenolic acid & VCEAC values (21) \\
\hline Ferulic acid & 170.5 \\
$p$-Coumaric acid & 186.0 \\
Syringic acid & 80.4 \\
Caffeic acid & 103.3 \\
$p$-Hydroxybenzoic acid & 4.8 \\
\hline
\end{tabular}

(14), the enhancement of $15 \mathrm{ADON}$ accumulation observed in supplemented cultures was consistent with the activating effect ascribed to hydrogen peroxide by Ochiai et al. (27) and Ponts et al. (31). According to the publication of Amorati et al. (2), the radical scavenging efficiency of phenolic acids is only consistent for $\mathrm{pH}$ values higher than their $\mathrm{pKa}$ (usually close to 4.5). Therefore, we could reasonably assume that the opposite effect of ferulic acid observed in this study and in the one of Boutigny et al. (9) was linked to differences in $\mathrm{pH}$ values of the culture media. Actually, in the minimal medium used by Boutigny et al. (9), the $\mathrm{pH}$ value, initially close to 6.5 , dramatically decreased as early as $24 \mathrm{~h}$ after fungal inoculation to attain a value close to two in 3-day-old cultures (25). In the GYEP cultures performed in this study, the $\mathrm{pH}$ value also decreases rapidly but its value remains $>4.5$ until the end of the cultures (data not shown). In a general manner, antioxidant compounds have already been involved in modulation of toxin production such as aflatoxins (29). According to Passone et al. (29), antioxidant compounds may act via a nonspecific mechanism, involving the perturbation of membrane function and leading to a modification of its permeability. In addition, antioxidant activity of phenolic acids was suggested to play an important part in their ability to regulate gene expression (37). Our results supported the previous conclusion. Accumulation of TRI5 transcripts was modulated by phenolic acid supplementation, enhanced in the presence of a high antioxidant compound such as ferulic acid and repressed in the presence of a weak antioxidant phenolic acid such as $p$-hydroxybenzoic acid. These data support the previous conclusion of Boutigny et al. (9), indicating that modification of trichothecenes biosynthesis by ferulic acid could be regulated at the transcriptional level.

However, BHT-associated data, with regard to $F$. graminearum strain CBS185.32, do not fit into this correlation. The BHT VCEAC value is 77.4 (21). Although this VCEAC value is lower than the VCEAC values of ferulic acid or $p$-coumaric acid (Table 3 ), BHT activates DON+15ADON accumulation 24 times (compared with 11 and 20 times for ferulic acid and $p$-coumaric acid, respectively). These data indicate that, even if antioxidant capacities seem to be important properties of the phenolic compounds that influence their ability to interact with DON+15ADON accumulation, they are not sufficient to account for all the observed trends. Because BHT is very lipophilic (highly alkylated), it could be hypothesized that its transfer rate into cells may be high enough to compensate for the lower antioxidant capacity compared with ferulic acid.

This study showed that antioxidant properties of phenolic acids may, in part, explain important effects on DON and 15ADON production by $F$. graminearum. This effect may partially be ascribed to a transcriptional control of the TRI5 gene. It should be noticed that the $0.5 \mathrm{mmol}$ liter $^{-1}$ concentration of phenolic acids that was used throughout the study is compatible with levels of ferulic and $p$-coumaric acid that are commonly found in corn kernels (7). Thus, in planta, according to the in vitro modulation of TCTB biosynthesis associated with phenolic acids, different compositions in phenolic acids of kernels from various cultivars of cereals may reflect different degrees of sensitivity to mycotoxinogenesis. This hypothesis, which strongly fits in with the results of Hamzehzarghani et al. (17), is currently being investigated.

\section{ACKNOWLEDGMENTS}

These results are part of N. Ponts' Ph.D. project, financially supported by the Conseil Régional de la Région Aquitaine and the Institut National de la Recherche Agronomique.

\section{LITERATURE CITED}

1. Aldred, A., and Magan, N. 2004. Prevention strategies for trichothecenes. Toxicol. Lett. 153:165-171. 
2. Amorati, R., Pedulli, G. F., Cabrini, L., Zambonin, L., and Landi, L. 2006. Solvent and $\mathrm{pH}$ effects on the antioxidant activity of caffeic and others phenolic acids. J. Agric. Food Chem. 54:2932-2937.

3. Antolovich, M., Bedgood, D. R., Bishop, A. G., Jardine, D., Prenzler, P. D., and Robards, K. 2004. LC-MS investigation of oxidation products of phenolic antioxidants. J. Agric. Food Chem. 52:962-971.

4. Asiegbu, F. O. 2000. Effects of carbohydrates, ethanol and selected cell wall phenolics on in vitro growth of necrotrophic fungi-Heterobasidion annosum and Fusarium avenaceum. J. Basic Microbiol. 40:139-148.

5. Bakan, B., Bily, A. C., Melcion, D., Cahagnier, B., Regnault-Roger, C., Philogene, B. J., and Richard-Molard, D. 2005. Possible role of plant phenolics in the production of trichothecenes by Fusarium graminearum strains on different fractions of maize kernels. J. Agric. Food Chem. 51:2826-2831.

6. Beekrum, S., Govinden, R., Padayachee, T., and Odhav, B. 2003. Naturally occurring phenols: A detoxification strategy for fumonisin B1. Food Addit. Contam. 20:490-493.

7. Bily, A. 2003. Rôle et importance des déhydrodimères d'acide férulique et autres phénylpropanoïdes dans les mécanismes de résistance de Zea mays L. à Fusarium graminearum Schwabe. Ph.D. dissertation, Université de Pau et des Pays de l'Adour, Pau, France.

8. Bolarinwa, A., and Linseisen, J. 2005. Validated application of a new high-performance liquid chromatographic method for the determination of selected flavonoids and phenolic acids in human plasma using electrochemical detection. J. Chromatogr. B 823:143-151.

9. Boutigny, A. L., Barreau, C., Atanasova-Penichon, V., Verdal-Bonnin, M. N., Pinson-Gadais, L., and Richard-Forget, F. 2009. Ferulic acid, an efficient inhibitor of type B trichothecene biosynthesis and Tri gene expression in Fusarium liquid cultures. Mycol. Res. 113:746-753.

10. Boutigny, A. L., Richard-Forget, F., and Barreau, C. 2008. Natural mechanisms for cereal resistance to Fusarium mycotoxins accumulation. A review. Eur. J. Plant Pathol. 121:411-423.

11. Chipley, J. R., and Uraih, N. 1980. Inhibition of Aspergillus growth and aflatoxin release by derivatives of benzoic acid. Appl. Environ. Microbiol. 40:352-357.

12. D'Mello, J. P. F., Placinta, C. M., and MacDonald, A. M. C. 1999. Fusarium mycotoxins: a review of global implications for animal health, welfare and productivity. Anim. Feed Sci. Technol. 80:183-205.

13. Gardiner, D. M., Kazan, K., and Manners J. M. 2009. Nutrient profiling reveals potent inducers of trichothecene biosynthesis in Fusarium graminearum. Fungal Genet. Biol. 41:600-623.

14. Gil-ad, N. L., and Mayer A. M. 1999. Evidence for rapid breakdown of hydrogen peroxide by Botrytis cinerea. FEMS Microbiol. Lett. 176:455461.

15. Glass, N. L., and Donaldson, G. C. 1995. Development of primer sets designed for use with the PCR to amplify conserved genes from filamentous ascomycetes. Appl. Environ. Microbiol. 61:1323-1330.

16. Guiraud, P., Steiman, R., Seiglemurandi, F., and Benoitguyod, J. L. 1995. Comparison of the toxicity of various lignin-related phenolic-compounds toward selected fungi perfecti and fungi imperfecti. Ecotoxicol. Environ. Saf. 32:29-33.

17. Hamzehzarghani, H., Kushalappa, A.C., Dion, Y., Rioux, S., Comeau, A., Yaylayan, V., Marshall, W. D., and Mather, D. E. 2005. Metabolic profiling and factor analysis to discriminate quantitative resistance in wheat cultivars against Fusarium head blight. Physiol. Mol. Plant Pathol. 66:119-133.

18. Hua, S. S. T., Grosjean, O. K., and Baker, J. L. 1999. Inhibition of aflatoxin biosynthesis by phenolic compounds. Lett. Appl. Microbiol. 29:289-291.

19. Jandera, P., Skerikova, V., Rehova, L., Hajek, T., Baldrianova, L., Skopova, G., Kellner, V., and Horna, A. 2005. RP-HPLC analysis of phenolic compounds and flavonoids in beverages and plant extracts using a CoulArray detector. J. Sep. Sci. 28:1005-1022.
20. Jiao, F., Kawakami, A., and Nakajima, T. 2008. Effects of different carbon sources on trichothecene production and Tri gene expression by Fusarium graminearum in liquid culture. FEMS Microbiol. Lett. 285:212-219.

21. Kim, D. O., and Lee, C. Y. 2004. Comprehensive study on vitamin C equivalent antioxidant capacity (VCEAC) of various polyphenolics in scavenging a free radical and its structural relationship. Crit. Rev. Food Sci. Nutr. 44:253-273.

22. Kim, J. H., Yu, J., Mahoney, N., Chan, K. L., Molyneux, R. J., Varga, J., Bhatnagar, D., Cleveland, T. E., Nierman, W. C., and Campbell, B. C. 2008. Elucidation of the functional genomics of antioxidant-based inhibition of aflatoxin biosynthesis. Int. J. Food Microbiol. 122:49-60.

23. Kim, Y. M., Farrah, S., and Baney, R. H. 2007. Structure-antimicrobial activity relationship for silanols, a new class of disinfectants, compared with alcohols and phenols. Int. J. Antimicrob. Agents 29:217-222.

24. Kimura, M., Tokai, T., Takahashi-Ando, N., Ohsato, S., and Fujimara, M 2007. Molecular and genetic studies of Fusarium trichothecenes biosynthesis: pathways, genes, and evolution. Biosci. Biotechnol. Biochem. $71: 2105-2123$

25. Merhej, J., Boutigny, A. L., Pinson-Gadais, L., Richard-Forget, F., and Barreau, C. 2010. Acidic $\mathrm{pH}$ during in vitro culture is determinant for TRI genes expression and trichothecenes B biosynthesis in Fusarium graminearum. Food Addit. Contam. 16:1-8.

26. Miller, J. D., Young, J. C., and Trenholm, H. L. 1983. Fusarium toxins in field corn. I. Time course of fungal growth and production of deoxynivalenol and other mycotoxins. Can. J. Bot. 61:3080-3087.

27. Ochiai, N., Tokai, T., Takahashi-Ando N., Fujimura, M., and Kimura, M. 2007. Genetically engineered Fusarium as a tool to evaluate the effects of environmental factors on initiation of trichothecenes biosynthesis. FEMS Microbiol. Lett. 275:53-61.

28. Park, K. D., Park, Y. S., Cho, J. S., Sun, W. S., Kim, S. H., Jung, D. H., and Kim, J. H. 2004. Antimicrobial activity of 3-O-acyl-(-)-epicatechin and 3-o-acyl-(+)-catechin derivatives. Planta Med. 70:272-276.

29. Passone, M. A., Resnik, S. L., and Etcheverry, M. G. 2005. In vitro effect of phenolic antioxidants on germination, growth and aflatoxin B accumulation by peanut Aspergillus section Flavi. J. Appl. Microbiol. 99:682-691.

30. Pinson-Gadais, L., Richard-Forget, F., Frasse, P., Barreau, C., Cahagnier, B., Richard-Molard, D., and Bakan, B. 2008. Magnesium represses trichothecenes biosynthesis and modulates Tri5, Tri6, and Tri12 genes expression in Fusarium graminearum. Mycopathologia 165:51-59.

31. Ponts, N., Pinson-Gadais, L., Barreau, C., Richard-Forget F., and Ouellet, T. 2007. Exogenous $\mathrm{H}_{2} \mathrm{O}_{2}$ and catalase treatments interfere with Tri genes expression in liquid cultures of Fusarium graminearum. FEBS Lett. 581:443-447.

32. Ponts, N., Pinson-Gadais, L., Verdal-Bonnin, M. N., Barreau, C., and Richard-Forget, F. 2006. Accumulation of deoxynivalenol and its 15acetylated form is significantly modulated by oxidative stress in liquid cultures of Fusarium graminearum. FEMS Microbiol. Lett. 258:102-107.

33. Reid, L. M., Mather, D. E., Arnason, J. T., Hamilton, R. I., and Bolton, A. T. 1992. Changes in Phenolic Constituents of Maize Silk Infected with Fusarium graminearum. Can. J. Bot. 70:1697-1702.

34. Rice-Evans, C. A., Miller, N. J., and Paganga, G. 1996. Structureantioxidant activity relationships of flavonoids and phenolic acids. Free Radic. Biol. Med. 20:933-956.

35. Rios, G., Pinson-Gadais, L., Abecassis, J., Zakhia-Rozis, N., and LullienPellerin, V. 2009. Assessment of dehulling efficiency to reduce deoxynivalenol and Fusarium level in durum wheat grains. J. Cereal Sci. 49:387-392.

36. Wallace, G., and Fry, S. C. 1994. Phenolic components of the cell plant wall. Int. Rev. Cytol. 151:229-267.

37. Yeh, C. T., and Yen, G. C. 2003. Effects of phenolic acids on human phenolsulfotransferases in relation to their antioxidant activity. J. Agric. Food Chem. 51:1474-1479. 